\title{
Short communication: Milk fat globule membrane as a potential delivery system for liposoluble nutrients
}

\author{
J.-B. Bezelgues, ${ }^{1}$ F. Morgan, ${ }^{2}$ G. Palomo, ${ }^{2}$ L. Crosset-Perrotin, and P. Ducret \\ Nestlé Research Center, Department of Food Science and Technology, Lausanne, Switzerland
}

\begin{abstract}
A soft physical process was used to extract and purified bovine milk fat globule membrane (MFGM) fractions on a pilot scale. Oil-in-water emulsions enriched with $\alpha$-tocopherol and lycopene were then prepared and stabilized with the extracted MFGM fraction and conventional milk protein concentrates (i.e., whey proteins, caseinate). A protocol of in vitro digestion was set up to evaluate the bioaccessibility of the tocopherol and lycopene in the different emulsions. Bioaccessibility was defined as the capacity of liposoluble compounds to be transferred into mixed micelles formed during the digestion process. Results showed that the accumulation of the tocopherol and lycopene into mixed micelles in MFGM-stabilized emulsions was around 2-fold greater than in emulsions stabilized with conventional milk proteins. This result confirms the potential use of MFGM-enriched ingredients as delivery systems of liposoluble nutrients in food formulations.
\end{abstract}

Key words: milk fat globule membrane, lycopene, tocopherol, mixed micelle

During the last decades, the development of dairy technology has permitted a better valorization of milk components and today, increasing numbers of new dairy ingredients claiming nutritional and functional benefits are found in the marketplace. More particularly, specific minor fractions of milk such as milk fat globule membrane (MFGM) represent an interesting and valuable natural material with potential textural and bioactive properties (German and Dillard, 2006; Singh, 2006; Bertram et al., 2007), which could be used in many food applications.

In milk, the lipid phase (generally around $40 \mathrm{~g} / \mathrm{L}$ ) exists as an emulsion of oil droplets dispersed in the aqueous phase (Danthine et al., 2000; Jensen, 2002). This fat dispersion takes the form of finely divided spheres $(0.1-15 \mu \mathrm{m})$ surrounded by a 4 - to $10-\mathrm{nm}$ multilayer membrane composed primarily of triglycerides,

Received September 17, 2008.

Accepted November 6, 2008.

${ }^{1}$ Corresponding author: jean-baptiste.bezelgues@rdls.nestle.com

${ }^{2}$ Current address: Lactalis R\&D, 35240 Retiers, France. proteins, and phospholipids, which is referred as the MFGM (McPherson et al., 1984; Danthine et al., 2000). This multilayered chemical complex forms a quantitatively small but functionally and nutritionally very important fraction because of the presence of polar lipids and specific glycoproteins (Spitsberg, 2005).The concentration of MFGM in milk varies from 0.4 to 1.5 $\mathrm{g} / \mathrm{L}$ according to published results (Jensen, 2002).

Besides its natural composition, which can be seen as a functional mix of bioactive lipids and proteins (Van Hooijdonk et al., 2000; Spitsberg, 2005), MFGM is the native emulsifier in milk, and the phospholipid-protein layer acts as a surfactant to prevent coalescence and flocculation of the fat globules in their aqueous environment. The surface active properties of MFGM may play a role in the transport and delivery of liposoluble nutrients along the gastrointestinal tract (Liu et al., 1995).

Fat-soluble nutrients such as $\alpha$-tocopherol and carotenoids (e.g., lycopene) have beneficial effects in human health particularly in protection against cancer, cardiovascular diseases, and age-related diseases (Julianto et al., 2000; Reboul et al., 2006) and their bioavailability is influenced by various factors such as food matrix, formulation, and process conditions. The mechanisms that might explain the low and variable absorption of carotenoids, for instance, are not yet well understood (Borel et al., 2000) because the fate of these nutrients in the gastrointestinal tract is still unclear. The uptake of these nutrients by the body should start during digestion and requires their solubilization into mixed micelles formed with bile salts in the lumen of the duodenum (Traber et al., 1990; Garrett et al., 1999; Julianto et al., 2000). Therefore, the measurement of the accumulation of these lipophilic molecules into the micellar phase using an in vitro digestion model could be useful to estimate their bioaccessibility (Garrett et al., 1999; Tyssandier et al., 2003).

In a previous study on vitamin $\mathrm{D}_{3}$ absorption, Liu et al. (1995) proposed that the transfer to mixed micelles and the absorption of vitamin $\mathrm{D}_{3}$ is enhanced because of the beneficial interaction of MFGM-stabilized microdispersions with bile salts. In this study (Liu et al., 1995), it was concluded that the interaction of MFGM 


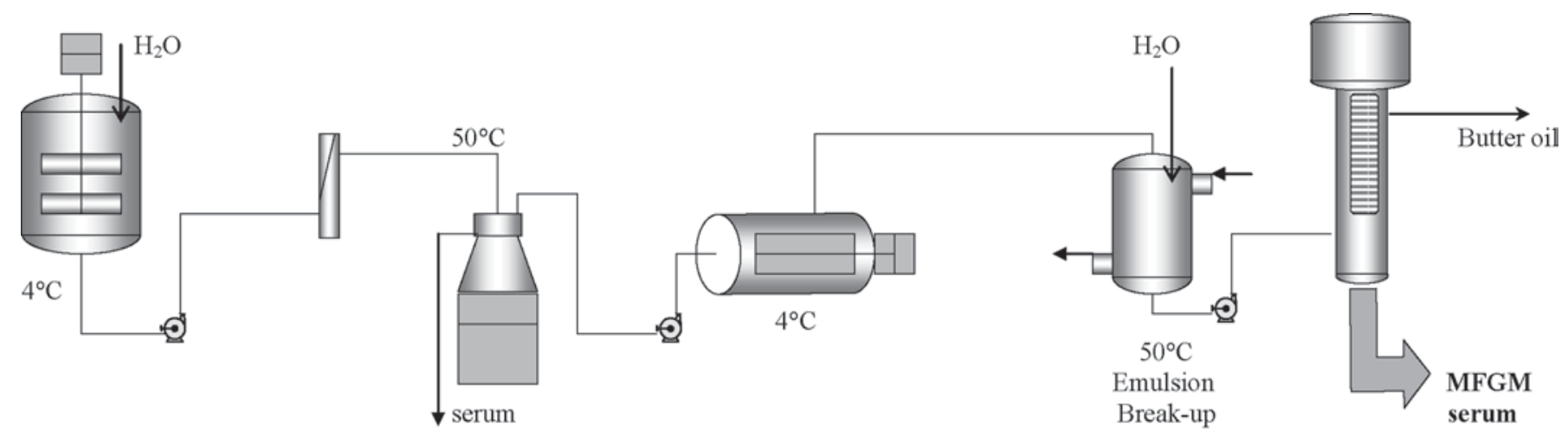

Washing and Concentration (centrifugation)
Maturation: crvstallization

(scraped surface heat exchanger)
MFGM Extraction

(centrifugation)

Figure 1. Preparation of purified milk fat globule membrane (MFGM) extract on a pilot scale.

with enzymes and bile salts that takes place in the lumen of the intestine should be the key element for the enhancement of the intestinal absorption of vitamin $\mathrm{D}_{3}$. Enhancement of the intestinal adsorption of vitamin A has been also reported in emulsions stabilized with MFGM extracts (Moriwaki et al., 1990). Therefore, MFGM extracts should be applicable in healthier food products as natural adsorption promoter for liposoluble vitamins and antioxidants (Liu et al., 1995).

The purpose of this work was to provide some insight on the potential use of MFGM extracts as lymphatic adsorption promoter for lipophilic nutrients such as tocopherol or lycopene. Oil-in-water emulsions stabilized with purified MFGM or conventional dairy proteins were tested in an in vitro digestion model. Fresh whole milk was purchased from a local dairy cooperative (Cremo, Mont sur Lausanne, Switzerland). The milk was not heat-treated before MFGM extraction. Purified MFGM fractions were extracted on a pilot scale using our own process, which is described in Figure 1.

In brief, fresh milk was concentrated up to $80 \%$ fat using a bowl disc centrifuge at $50^{\circ} \mathrm{C}$. The concentrated cream was washed with 5 volumes of salty water $(0.15$ $\mathrm{mol} / \mathrm{L}$ sodium chloride) under gentle stirring at $50^{\circ} \mathrm{C}$ and then reconcentrated up to $80 \%$ fat. The washed and concentrated cream was then matured at $4^{\circ} \mathrm{C}$ using a scraped surface heat exchanger Votator (ChemTech, Reading, UK) and then destabilized by heating at $50^{\circ} \mathrm{C}$. The destabilized emulsion was centrifuged at 5,000 $\times g$ to separate the serum containing the MFGM fragments and the oil phase. The serum was finally pasteurized and freeze-dried.

Sodium caseinate and whey protein isolate (Prolacta 90) were provided by Lactalis (Retiers, France). $\alpha$-Tocopherol and lycopene (oleoresin) were purchased respectively from Roche Vitamins Ltd. (Basel, Swit- zerland) and Indena (Milan, Italy). Pepsin, pancreatin (porcine), bile extract (porcine), and sodium taurocholate were purchased from Sigma Chemical Co. (St Louis, MO).

Portions of dried emulsifiers (purified MFGM, whey protein, sodium caseinate) were resuspended in PBS at $\mathrm{pH}$ 7. A mixture of medium-chain triglycerides (MCT) and triolein $(80 / 20)$ was added $(100 \mathrm{~g} / \mathrm{kg}$ final emulsion) to the aqueous suspension of emulsifier and dispersed using a high-pressure homogenizer at $50^{\circ} \mathrm{C}$. A first homogenization stage was done at $350 \times 10^{5} \mathrm{~Pa}$ and a second at $75 \times 10^{5} \mathrm{~Pa}$. Optimization of emulsifier concentration was done by measuring the particle size distribution in the different emulsions.

The nitrogen content of MFGM fractions was determined using the Kjeldahl method. Qualitative protein profile was done by SDS-PAGE. Polar lipids were extracted using a 3-step cold extraction procedure. The sample was mixed vigorously with $\mathrm{CHCl}_{3}-\mathrm{MeOH}$ (2:1, $\mathrm{vol} / \mathrm{vol}$ ) and $\mathrm{KCl}$ solution (1\%), followed by centrifugation at $3,000 \times g$ for $10 \mathrm{~min}$. The organic phase was collected and the residue was reextracted twice more with $\mathrm{CHCl}_{3}-\mathrm{MeOH}(2: 1, \mathrm{vol} / \mathrm{vol})$. The 3 organic phases were collected, dried in a rotary evaporator, and resuspended in $\mathrm{CHCl}_{3}-\mathrm{MeOH}(9: 1, \mathrm{vol} / \mathrm{vol})$. This solution was filtered through a membrane filter $(0.45 \mu \mathrm{m})$ and further analyzed. The analysis and quantification of the phospholipids was then carried out on a HPLC silica column (Prevail silica, 3 micron $150 \times 3 \mathrm{~mm}$, Agilent, Morges, Switzerland) with a gradient elution using chloroform-methanol and triethylamine at $\mathrm{pH}: 3$. The detection was performed with an evaporative light scattering detector at $45^{\circ} \mathrm{C}$.

The standard procedure to study the efficiency of MFGM to be used as delivery system for liposoluble compounds (tocopherol, lycopene) was adapted from 
4.5 ug loaded
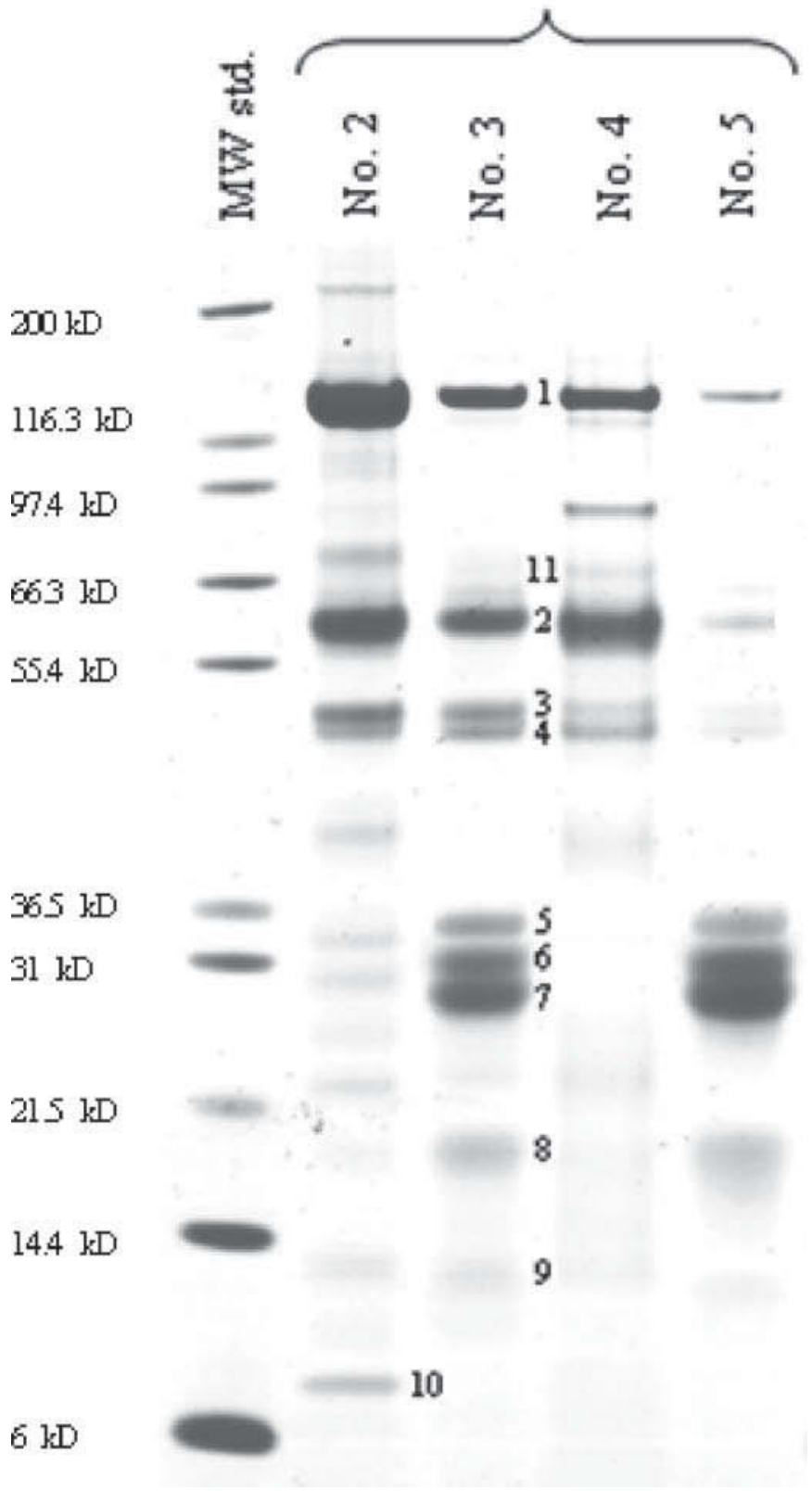

Figure 2. Sodium dodecyl sulfate-PAGE (12\% bis-tris gel) patterns of milk fat globule membrane (MFGM) isolate. Lane no. $1=$ molecular weight marker; lane no. $2=$ washed MFGM isolate, batch 1; lane no. $3=$ nonwashed MFGM; lane no. $4=$ washed MFGM batch 2 ; lane no. $5=$ commercial butter serum. Protein identification: $1=$ xanthine oxidase; $2=$ butyrophilin; $3=$ adipohilin; $4=$ lactadherin; $5,6,7=$ caseins; $8=\beta$-LG; $9=\alpha$-LA; $10=$ fatty acid bound protein (identification not 100\%); $11=$ CD36 (identification not 100\%).

that described by Garrett et al. (1999). This approach consists of measuring the accumulation of the bioactive molecules into mixed micelles, which is the colloidal structure that defines the adsorption in the small intestine (Garrett et al., 1999). Briefly, $35 \mathrm{~g}$ of model emul-
Table 1. Composition of the purified milk fat globule membrane (MFGM) fraction

\begin{tabular}{lc}
\hline Composition & Purified MFGM fraction \\
\hline Total protein (\%) & 55 \\
Total lipid (\%) & 37.9 \\
Triglycerides (\%) & 10 \\
Phospholipids (\%) $^{1}(\%)$ & 25 \\
Phospholipid profile & \\
SM & 26.80 \\
PC & 22.45 \\
PE & 26.95 \\
PS & 13 \\
PI & 7.30 \\
PA & 3.45 \\
\hline
\end{tabular}

${ }^{1} \mathrm{SM}=$ sphingomyelin; $\mathrm{PC}=$ phosphatidylcholine; $\mathrm{PE}=$ phosphatidylethanolamine; PS = phosphatidylserine; $\mathrm{PI}=$ phosphatidylinositol; $\mathrm{PA}=$ phosphatidic acid.

sion is cooled on ice before acidification $(\mathrm{pH} 2)$ with 1 $M \mathrm{HCl}$ and the addition of $2 \mathrm{~mL}$ of porcine pepsin (40 $\mathrm{mg} / \mathrm{mL}$ in $0.1 \mathrm{M} \mathrm{HCl}$ ). The mixture is transferred to a clean amber vial and incubated at $37^{\circ} \mathrm{C}$ in a water bath shaking at $120 \mathrm{rpm}$ for $1 \mathrm{~h}$. Then, the $\mathrm{pH}$ of the partially digested emulsion was increased to 5.3 by adding 1 to $1.5 \mathrm{~mL}$ of $0.9 \mathrm{M}$ sodium bicarbonate followed by the addition of a mixture of bile extract and pancreatin $(9 \mathrm{~mL}$ containing $2 \mathrm{mg} / \mathrm{mL}$ pancreatin and 12 $\mathrm{mg} / \mathrm{mL}$ bile extract in $100 \mathrm{mmol} / \mathrm{L}$ sodium bicarbonate solution). The final concentrations of pancreatin and bile extract in the mixture were 0.4 and $2.4 \mathrm{mg} / \mathrm{mL}$, respectively. The $\mathrm{pH}$ was increased to 7.5 by adding 1 $M$ sodium hydroxide. The partially digested emulsion was incubated in the water bath under stirring at $37^{\circ} \mathrm{C}$ for $2 \mathrm{~h}$ to complete the intestinal phase of the in vitro digestion.

The aqueous fraction that contained micelles was separated from residual oil droplets and particles of digesta by ultracentrifugation (Beckman) at 165,000 $\times g$ at $4^{\circ} \mathrm{C}$ for $95 \mathrm{~min}$. The aqueous phase fraction (micellar fraction) was collected and passed through a filter with $0.22-\mu \mathrm{m}$ pores (Milian, Gahanna, $\mathrm{OH}$ ). Tocopherols were then extracted with hexane from the micellar phase and quantified by HPLC coupled with a fluorimeter. Lycopene was extracted with hexane from the micellar phase and quantified by spectrophotometry at $470 \mathrm{~nm}$. The accumulation of liposoluble nutrients in mixed micelles is expressed as the ratio between the quantity measured in the micellar phase and the initial concentration in the emulsion.

The Nu-PAGE patterns depicted in Figure 2 confirmed that our process facilitated the removal of caseins and whey proteins and the concentration of MFGM proteins such as xanthine oxidase, butyrophilin, and lactadherin. Transmission electron microscopy analysis confirmed that the washed MFGM fraction contained fewer casein micelles than the crude extract and that 


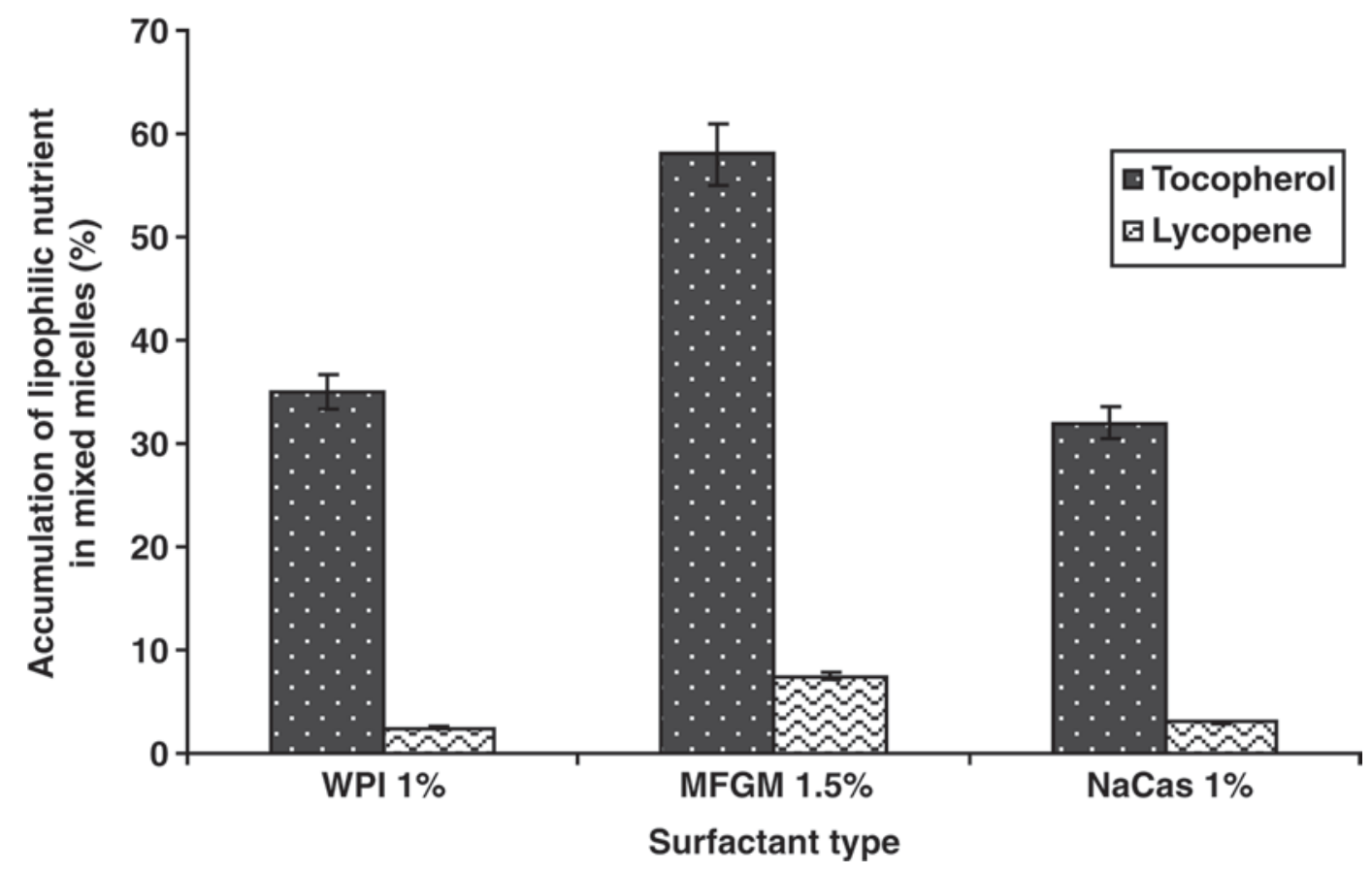

Figure 3. Percentage of accumulation of tocopherol and lycopene in mixed micelles after in vitro digestion of different model emulsions. WPI = whey protein isolate; MFGM = milk fat globule membrane; NaCas = sodium caseinate.

fragments of membrane were present. As described in the Table 1, this extract was also enriched in polar lipids, and the phospholipid profile was similar to that of bovine milk. As shown in Tables 1 and 2, all emulsions had a fine and close mean particle diameter.

We observed, as depicted in Figure 3, that the accumulation of $\alpha$-tocopherol in mixed micelles in the emulsion stabilized by MFGM extract was around 2 -fold higher than that of conventional milk proteins (Table 2).

Garrett et al. (1999) observed a very low micellarization of lycopene during the digestion of food, suggesting a low bioavailability. In our models, the transfer rate of lycopene in the micellar phase was still low but greatest (around 2-fold) for the emulsion stabilized with MFGM-derived material (Table 3).
Interestingly, these in vitro experiments confirm the potential of the MFGM-enriched fraction to increase the micellarization of liposoluble nutrients, suggesting that the bioaccessibility of such molecules could be enhanced in low-fat food systems. The particular composition of MFGM, which is a mix of glycoproteins and complex polar lipids, could explain its properties during the solubilization of lipophilic species in mixed micelles. More particularly, the MFGM lipids, which are partially hydrolyzed by lipases during digestion, may form specific mesophases (vesicules, lamellar phases) that can enhance the solublization rate in the micellar phase compared with conventional emulsifiers. The presence of phospholipids in the emulsion stabilized by the MFGM isolate could explain the difference observed (Liu et al., 1995).

Table 2. Composition and mean particle diameter (D3,2; Sauter diameter) of the emulsions enriched with tocopherol (toco) ${ }^{1}$

\begin{tabular}{lccc}
\hline Composition $(\%)$ & Toco/MFGM pure & Toco/WPI & Toco/NaCas \\
\hline Medium-chain triglycerides/triolein $(80 / 20)$ & 10.00 & 10.00 & 10.00 \\
Emulsifier & 1.50 & 1.00 & 1.00 \\
Tocopherol & 0.020 & 0.020 & 0.020 \\
Water & 88.48 & 88.98 & 88.98 \\
Total phospholipid & 0.35 & 0.00 & 0.00 \\
Total protein & 0.82 & 0.88 & 0.85 \\
D3,2 $(\mu \mathrm{m})$ & 0.76 & 0.65 & 0.72 \\
\hline
\end{tabular}

${ }^{1}$ Emulsions: $\mathrm{MFGM}=$ milk fat globule membrane; WPI = whey protein isolate; $\mathrm{NaCas}=$ sodium caseinate. 
Table 3. Composition and mean particle diameter (D3,2; Sauter diameter) of the emulsions enriched lycopene $(\text { lyco })^{1}$

\begin{tabular}{lccc}
\hline Composition (\%) & Lyco/MFGM pure & Lyco/WPI & Lyco/NaCas \\
\hline Medium-chain triglycerides/triolein $(80 / 20)$ & 10.00 & 10.00 & 10.00 \\
Emulsifier & 1.50 & 1.00 & 1.00 \\
Lycopene & 0.010 & 0.010 & 0.010 \\
Water & 88.50 & 89 & 89 \\
Total phospholipid & 0.32 & 0.00 & 0.00 \\
Total protein & 0.80 & 0.85 & 0.80 \\
D3,2 $(\mu \mathrm{m})$ & 0.50 & 0.60 & 0.50 \\
\hline
\end{tabular}

${ }^{1}$ Emulsions: $\mathrm{MFGM}=$ milk fat globule membrane; $\mathrm{WPI}=$ whey protein isolate; $\mathrm{NaCas}=$ sodium caseinate.

The results of this study suggest that the difference between the promotion effects of absorption of $\alpha$-tocopherol and lycopene by the purified MFGM fraction and conventional dairy proteins may be due to the formation of MFGM compounds-bile salt mixed micelles. These preliminary in vitro experiments should be repeated in a more complex matrix to more closely simulate a real food matrix. It should be interesting to further characterize the mesophases that are formed in the micellar phase. Moreover, specific butter serums that are now commercially available should be tested and compared with the purified MFGM fraction. Indeed, in these commercial fractions, the presence of caseins may completely change the properties of MFGM toward micellarization.

These preliminary in vitro assays with low-fat model emulsions have confirmed the ability of MFGM-purified fraction to increase the transfer rate of liposoluble bioactive molecule into bile salts micelles. This observation suggests that there may be possible nutritional applications as lymphatic absorption promoters in low-fat food products.

\section{REFERENCES}

Bertram, Y. F., C. S. Norris, and A. K. H. MacGibbon. 2007. protein and lipid composition of bovine milk-fat -globule memebrane. Int. Dairy J. 17:275-288.

Danthine, S., Ch. Blecker, M. Paquot, N. Innocente, and C. Deroanne. 2000. Evolution des connaissances sur la memebrane du globule gras du lait: Synthèse bibliographique. Lait 80:209-222.

Garrett, D. A., M. L. Failla, and R. J. Sarama. 1999. Develpment of an in vitro digestion method to assess carotenoid bioavailability from meals. J. Agric. Food Chem. 47:4301-4309.
German, J. B., and C. J. Dillard. 2006. Composition, structure and absorption of milk lipids: A source of energy, fat soluble nutrients and bioactives molecules. Crit. Rev. Food Sci. Nutr. 46:57-92.

Jensen, R. G. 2002. Invited review: The composition of bovine milk lipids. J. Dairy Sci. 85:295-350.

Julianto, T., K. H. Yuen, and A. M. Noor. 2000. Improved bioavailability of vitamin $\mathrm{E}$ with a self emulsifying formulation. Int. J. Pharmaceut. 200:53-57.

Liu, H. X., I. Adachi, I. Horikoshi, and M. Ueno. 1995. Mechanism of promotion of lymphatic grig absorption by milk fat globule memebrane. Int. J. Pharmaceut. 118:55-64.

McPherson, A. V.. M. C. Dash, and B. J. Kitchen. 1984. Isolation of bovine milk fat globule membrane material form cream without prior removal of caseins and whey proteins. J. Dairy Res. 51:113121.

Moriwaki, T., H. Yoshikawa, K. Takada, and S. Muranishi. 1990. DDS with milk fat globule membrane: Gastrointestinal absorption of vitamin A acetate. Yakuzaikaku 50:263-267.

Reboul, E., M. Richelle, E. Perrot, C. Desmoulins-Mazelet, V. Pirisi, and P. Borel. 2006. Bioaccessibility of carotenoids and vitamin E form their main dietary sources. J. Agric. Food Chem. 54:87498755 .

Singh, H. 2006. The milk fat globule membrane - A biophysical system for food applications. Curr. Opin. Colloid Interface Sci. 11:154163.

Spitsberg, V. L. 2005. Invited review: Bovine milk fat globule membrane as a potential nutraceutical. J. Dairy Sci. 88:2289-2294.

Traber, M.G., I. Golberg, E. Davidson, N. Lagmay, and H. J. Kayden. 1990. Vitamin E uptake by human intestinal cells during lipolysis in vitro. Gastroenterology 98:96-103.

Tyssandier, V., E. Reboul, J. F. Dumas, C. Bouteloup-Demange, M. Armand, J. Marcand, M. Sallas, and P. Borel. 2003. Processing of vegetable-bormne carotenoids in the human stomach and duodenum. Am. J. Physiol. Gastrointest. Liver Physiol. 284:913923.

Van Hoojidonk, A. C. M., K. D. Kussendrager, and J. M. Steijns. 2000. In vivo antimicrobial and antiviral activity of components in bovine milk and clostrum involved in non-specific defence. Br. J. Nutr. 84(Suppl. 1):S127-S134. 